\title{
Figures et dynamiques de la professionnalisation des communicateurs
}

Un miroir tendu aux associations en Belgique, en
France et Canada

\author{
Dany Baillargeon, professeur, \\ Université de Sherbrooke, \\ dany.baillargeon@usherbrooke.ca \\ Vincent Brulois, maître de conférence, \\ Université Paris 13, PRES Sorbonne Paris Cité, \\ LabSIC, brulois@sic.univ-paris13.fr \\ Catherine Coyette, assistante de recherche, \\ Université catholique de Louvain, LASCO, \\ catherine.coyette@uclouvain-mons.be \\ Marc D. David, professeur, \\ Université de Sherbrooke, \\ marc.d.david@usherbrooke.ca \\ François Lambotte, professeur, \\ Université catholique de Louvain, LASCO, \\ francois.lambotte@uclouvain-mons.be \\ Valérie Lépine, maître de conférences, \\ Université de Grenoble, GRESEC, \\ valerie.lepine@iut2.upmf-grenoble.fr
}

Cette recherche menée dans l'espace francophone commun à la Belgique, à la France et au Canada analyse les dynamiques de professionnalisation et identifie les discours et actions performatives qui participent à construire une «figure» du communicateur professionnel. L'analyse inductive d'entrevues auprès de dix-sept représentants actuels et passés de neuf associations professionnelles circonscrit trois formes que prend la figure du professionnel en communication : les valeurs (éthos), les actions (praxis) et les objets (artéfacts). La mise en relation des éléments qui composent ces formes permet d'identifier trois tensions : 1) l'aspiration à une professionnalisation déontique et stratégique pas toujours réalisée; 2) l'idéal véhiculé par les associations et une constante polarisation vers la tâche des professionnels; 3) un apparent débalancement entre les artéfacts normatifs et leur réelle performativité dans la praxis et dans l'éthos. Enfin, même si elle n'offre pas d'explication à ces tensions, cette recherche propose un outil méthodologique qui pourrait servir d'outil d'aide à la mise en place d'une politique professionnelle associative. 
Cette contribution s'inscrit dans le cadre d'une recherche collective et internationale menée dans l'espace francophone commun à la Belgique, à la France et au Canada. Fondée collectivement dans une démarche partenariale orientée vers les praticiens de la communication, elle constitue une invitation à engager l'analyse dynamique d'une professionnalisation disputée par de multiples instances représentatives, sur différents modes rhétoriques (juridique, déontologique, militant, casuistique...) et de services proposés aux professionnels (édition, événements, formation, conseil...).

Elle a pour objectif d'analyser les dynamiques de professionnalisation, à travers les instances représentatives de sept associations, et ainsi d'identifier les discours et actions performatives de figures du communicant professionnel : "Sensemaking begins with a self-conscious sensemaker » (Weick, 1995, p.22). Étymologiquement, la notion de «figure » signifie «quelque chose qui est fabriquée » qui nécessite donc un travail de production, de façonnage (Vasquez, Jolivet, 2011, p. 130). Pour exister, la figure doit être rendue présente, sa mise en présence lui permet ensuite d' « opérer », de devenir une «forme d'agentivité (...) présentée - ou re-présentée dans le discours » (Vasquez, Jolivet, 2011, p. 130). Par figures, nous comprenons donc les diverses conceptualisations de ce qu'est le professionnel de la communication, telles qu'elles sont rendues présentes (Cooren, 2010b) dans le discours des représentants d'associations. En d'autres termes, la «figure» du professionnel de la communication est mobilisée lorsque les représentants d'associations, dans le cas qui nous concerne, font intervenir des intérêts et des ordres spécifiques et locaux : "Autrement dit, si les textes bel et bien écrivent l'organisation, il faut alors montrer comment une telle "écriture" (au sens derridien du terme, impliquant donc la possibilité d'une écriture orale) peut aussi se faire à distance, à travers les effets de représentation et d'incarnation qui s'opèrent dans les interactions humaines. » Cooren (2010c, p. 29).

Nous défendons donc l'hypothèse que l'interprétation de la professionnalisation proposée par les représentants et porte-parole de chacune des associations reflète les formes d'engagement et d'action dans la sphère professionnelle de ces acteurs associatifs. Bien qu'elles n'attestent pas de l'effectivité de cet engagement - ce n'est pas parce que les associations dictent une conduite que cette dernière trouvera écho chez les praticiens - le discours sur la professionnalisation des représentants témoigne déjà d'un vouloir être, tout en proposant une intelligibilité des actions professionnelles de leurs membres. De plus, nous posons que cette professionnalisation se négocie à travers l'élaboration et la confrontation de figures professionnelles au sein des associations et entre les associations.

Il s'agit donc de renoncer - provisoirement - aux définitions ou cadres d'analyse de la professionnalisation a priori tels que la sociologie des professions les a forgés (Chapoulié, 1973; Abbot, 1988) et fait évoluer (Strauss, 1991; Dubar et Tripier, 
2005; Champy, 2011) de même qu'aux travaux traitant spécifiquement de la professionnalisation dans le champ des SIC (Brulois et Charpentier, 2010; de la Broise, 2006; de la Broise et al., 2008; Huet et de la Broise, 2010) pour laisser émerger les différentes figures ou représentations qui se complètent ou s'opposent dans les discours des associations représentatives, au plan national, des acteurs spécialisés dans la communication. Dans cette perspective, la démarche de la recherche est partiellement structurée par une hypothèse de travail, mais aussi largement ouverte à des analyses issues d'un recueil empirique de données dont la validité est assurée par un travail de triangulation des sources.

Afin de présenter avec la plus grande clarté cette démarche inductive et itérative originale, nous avons opté pour un déroulement du plan qui reflète les grandes étapes méthodologiques et intellectuelles de cette recherche.

\section{Un terrain d'enquête exploratoire auprès des associations professionnelles}

La méthodologie retenue repose sur un terrain d'enquêtes qualitatives dont le protocole est strictement commun aux chercheurs des trois pays impliqués de façon à pouvoir opérer une confrontation qui repose sur la prise en considération des contextes nationaux dans lesquels les champs professionnels (métiers, fonctions, compétences, rôles et représentations) ont émergé et se sont développés. Nos objectifs méthodologiques reposent ainsi sur l'identification des formes, des positionnements et postures adoptés par les acteurs de la représentation et de la défense corporative - associations et syndicats professionnels reconnus comme tels - à partir des discours de leurs porte-parole.

La stratégie méthodologique procède en quatre phases : 1) la conduite d'entrevues semi-dirigées; 2) l'identification des formes que prend la figure à travers un codage heuristique; 3) une confrontation matricielle des matériaux discursifs; 4) une cartographie des formes et des catégories donnant lieu à des grappes thématiques placées sur une carte perceptuelle pour mieux en saisir les relations. La première phase est décrite ci-après. Les autres phases seront décrites plus loin pour respecter le processus itératif derrière la construction de cette figure professionnelle.

Quatre grands segments et champs professionnels d'intervention des communicants ont été retenus pour l'étape exploratoire : les relations publiques et la communication institutionnelle, la communication publique et communication territoriale, la communication interne et sociale et la communication marketing. L'échantillon investigué représente donc, pour cette étape de la recherche, trois associations canadiennes (Association des agences de publicité du Québec : AAPQ; Forum des communicateurs gouvernementaux: FCG; Société québécoise des professionnels en relations publiques : SQPRP), une association belge (Association Belge de Communication Interne), cinq associations françaises (Association 
Française de Communication Interne, Syntec Conseil en Relations publiques, Communication \& Entreprise, Cap' Com et Communication Publique). Ces associations ont été choisies, car elles revendiquent une dimension corporative nationale dans les quatre grands champs professionnels retenus (communication marketing, relations publiques, communication interne et communicateurs gouvernementaux $)^{7}$.

Dix-sept entretiens approfondis (une à deux heures) auprès de représentants actuels et passés de ces associations ont été réalisés par des binômes de chercheurs, puis intégralement retranscrits pour l'analyse. Semi-directif, l'entretien explorait 1) les raisons d'être, motifs de positionnement et d'évolutions des associations; 2) les enjeux perçus et les modalités d'engagement de l'association dans la professionnalisation de leurs adhérents; 3) les rapports établis, attendus ou non, avec les acteurs impliqués dans la production et la diffusion de connaissances en SIC; 4) les trajectoires personnelles et institutionnelles ainsi que l'implication des représentants associatifs à l'égard de la reconnaissance mutuelle et publique des communicants membres de leur groupement.

Afin de se prémunir des biais d'une analyse qui reposerait sur le discours unique d'un représentant de l'association - fût-ce son président -, une triangulation des sources a été systématiquement opérée en réalisant une cueillette de données dissociées auprès de deux porte-parole légitimés, soit au moins deux entretiens disjoints: l'un avec le (ou la) président(e) en exercice, l'autre avec un ancien président ou le secrétaire général ou encore un membre du conseil d'administration chargé de mission par exemple. La troisième source est celle des productions éditoriales, symboliques et discursives, telles qu'elles sont disponibles sur les sites internet des associations, sans pour autant impliquer une analyse textuelle des sites en tant que tels. Cette phase d'enquête, déployée au printemps 2012, a été conduite grâce à la contribution de dix chercheurs belges, canadiens et français ${ }^{8}$ en sciences de l'information et de la communication.

\footnotetext{
${ }^{7}$ Cet échantillon raisonné construit pour la phase exploratoire de la recherche est non exhaustif. En France, d'autres associations ou syndicats répondent à ce critère de reconnaissance nationale et de représentativité corporative, telle que l'AACC ou l'ADEM pour n'en citer que deux. Dans une phase d'approfondissement, l'échantillon sera élargi et visera à représenter les acteurs locaux de la représentation (associations ou clubs de communicants en régions) ainsi que les représentants de professionnels liés à des secteurs d'activité spécifiques (Hôpital, Université par ex.)

${ }^{8}$ V. Brulois (U. de Paris 13), D. Chauvin (U. de Rennes 2), C. Coyette et F. Lambotte (U. catholique de Louvain), P. de la Broise (U. de Lille 3), V. Lépine et F. Martin-Juchat (U. de Grenoble), D. Baillargeon, Marc D. David (U. de Sherbrooke).
} 


\section{Cadrage théorique et construction de l'objet - Le concept de figure à partir de trois dimensions : l'éthos, la praxis et les artéfacts}

Suivant la posture de Cooren et d'autres auteurs mobilisant la notion de «figure», il convient de soutirer des matériaux discursifs les formes de cette figure. Ainsi, partant des matériaux discursifs nous avons opéré un premier niveau de codage heuristique ${ }^{9}$. Cela suppose d'analyser le discours des représentants associatifs comme une « instance de pratique sociale [...] dont les propriétés "organisantes" ont des impacts sur les actions et les comportements des gens » (Grant, Hardy, Oswick et Putnam, 2004, p.9). Plus précisément, nous avons entrepris de déplier le discours de sorte à faire émerger les valeurs que les représentants mobilisaient pour créer une « figure culturelle» (Cooren, 2010b) du professionnel.

L'articulation de la figure du professionnel de la communication semblait, dans le discours des représentants des associations, émerger sous trois formes, que nous avons nommées éthos, praxis et artéfacts.

\subsection{L'interprétation de la figure : l'éthos du professionnel}

En performant leur rôle (Trujillo, 1983), les représentants associatifs se trouvent animés des valeurs de leur profession en même temps qu'ils les animent à travers leur discours. Nous avons nommé cette première catégorie l'éthos, en référence à Geertz (1973) en cela qu'il représente une attitude évaluative, voire morale, en regard de la conception que le professionnel se fait de la réalité de sa profession. Pour reprendre Jorro (2009), les représentants associatifs chercheraient donc «à construire un positionnement professionnel adapté à l'idée qu'ils se font de la professionnalité du métier visé » (Jorro, 2009, p. 1). Cet éthos est avant tout réflexif : il engage le professionnel à rendre intelligible son attitude et ses actions en regard d'une conception préexistante du professionnel dans la communauté. «The ethos is made intellectually reasonnable by being shown to represent a way of life implied by the actual state of affairs which the world view describes [...]» (Geertz, 1973, p.127). En ce sens, l'éthos rend acceptables les actions du professionnel, intelligibles pour reprendre Trujillo, mais également socialement inscrites dans les rapports entre les acteurs. C'est pourquoi la figure émerge en partie du discours des représentants associatifs à travers un éthos évoqué du professionnel.

\footnotetext{
${ }^{9}$ Silvermann (2000) suggère que de façon à dégager du sens de matériaux qualitatifs, le chercheur construit des relations conceptuelles entre les contenus de discours d'abord en analysant ce qui est énoncé dans chaque segment de discours, puis en tentant de voir le liens entre les segments de l'ensemble des matériaux
} 


\subsection{L'action de légitimation de la figure : la praxis de légitimation.}

La seconde forme qu'a prise la figure est abordée sous forme d'inventaires des matériaux discursifs : nous avons recensé, dans le discours des représentants, les actions prises par les associations pour légitimer cette figure auprès des différentes instances (la haute direction, les parties prenantes, le public, etc.). Cette catégorie, nous l'avons appelée praxis. Notre définition de praxis englobe les gestes, les interventions, les prises de position, bref, les actions de l'association faites au nom d'une professionnalité du communicateur. Il faut donc voir ces actions comme des « pratiques », au sens des théories de l'acteur-réseau (ANT) (Latour, 1989 ; Callon, Latour et Akrich, 2006), qui produisent des relations entre des acteurs (les représentants/les parties prenantes) et les « enrôlent» dans le champ des dynamiques professionnelles. Ces mêmes pratiques agissent comme catalyseurs parce qu'elles engendrent des effets sur l'environnement, les gens et les choses. Elles enrôlent également d'autres acteurs. La praxis de légitimation revêt également l'idée d'excellence promue par la réalisation effective et constamment améliorée d'une action professionnelle. Pour MacIntyre, que citent Hesmondhalgh et Baker (2011, p.39), une pratique est :

[...] une forme de coopération humaine à travers laquelle des reconnaissances internes propres à cette activité se produisent dans la poursuite de standards d'excellence qui sont appropriés à [...] cette forme d'activité avec comme résultat que la capacité d'atteindre l'excellence et la reconnaissance de cette excellence sont constamment repoussées.

Pour les communicateurs, la professionnalisation peut être également reconnue dès lors qu'elle correspond à certains standards de qualité, institutionnalisés ou introjetés, qui les poussent à se dépasser pour les satisfaire. Ces normes ou ces standards, lorsqu'ils sont mobilisés en pratique, constituent un mode de reconnaissance entre pairs (internes), mais également aux yeux des parties prenantes (externes).

\subsection{Les symboles de légitimité : les artéfacts}

Enfin, nous avons procédé à un troisième inventaire (sur la base d'observations in situ, des discours et des sites internet): celui des objets qui agissent comme médiateurs, pour attester de la présence d'une figure professionnelle. Il peut donc s'agir de prix ou de labels de qualité remis aux praticiens pour l'excellence de leur travail, de codes de pratiques, d'études internes sur la profession, d'ouvrages de promotion des bonnes pratiques, etc. Aussi, nous avons recensé les différents objets 
portant l'agentivité de cette figure. Comme toute production culturelle, des objets, que nous avons appelés artéfacts, tiennent de lieu et place de la dynamique de professionnalisation. Ces symboles « résument, pour ceux qui y sont sensibles, ce qui est connu sur comment le monde est $[\ldots]$ et la façon dont ils doivent se comporter dans ce monde» (Geertz, 1973, p.127). Ces artéfacts cristallisent des « connaissances situées» (Caldwell, 2006) sur la profession et sont donc témoins des dynamiques produisant la figure du professionnel en même temps qu'ils participent à la construire. Par exemple, les revues professionnelles destinées aux membres «fonctionnent moins comme une tentative de parler au public au sens large [...] que comme une façon avec laquelle la communauté d'une industrie se parle à elle-même; produit du sens pour elle-même; se positionne et positionne ses membres. »(Caldwell, 2006, p. 133). Ainsi, ces artéfacts, par leur agentivité, influencent la conduite de la pratique professionnelle tout en étant médiateurs de l'éthos du professionnel.

\section{Une approche matricielle et dialogique des dynamiques de professionnalisation}

\section{1. Élaboration de la matrice}

Nous avions donc à la fois des matériaux culturellement construits - la figure du professionnel - et des matériaux formellement observables - les actions et les objets. Nous aurions pu choisir d'étudier qu'une seule de ces trois manifestations d'une dynamique professionnalisante. Or il nous apparaissait plus porteur de les mettre en rapport dialogique pour comprendre comment les tensions, voire les contradictions, entre l'éthos, la praxis et les artéfacts participent à cette dynamique. Autrement dit, cette professionnalisation est-elle consensuelle, donc ces dimensions pointent-elles dans la même « direction » ou sont-elles en constante négociation?

La troisième phase de notre stratégie méthodologique, après les entrevues, la création des trois catégories, consiste donc à mettre en rapport dialogique ces matériaux.

Martin (2002), dans sa construction d'une métathéorie sur la façon d'aborder les cultures organisationnelles, propose que les manifestations idéationnelles (notre éthos) et matérielles (notre praxis et nos artéfacts) peuvent être mises en relation, à l'intérieur de matrices, de façon à relever les discours en consensus (une culture intégrée et harmonieuse), en opposition (une culture de contestation) et en tensions (une culture d'ambiguité). Martin suggère d'utiliser une ou plusieurs matrices et mettre en relation les valeurs, les pratiques et les artéfacts pour déterminer les rapports consensuels, oppositionnels ou ambigus. Nous avons produit neuf matrices, soit une pour chaque association, que nous avons exemplifiées dans le Tableau 1. 


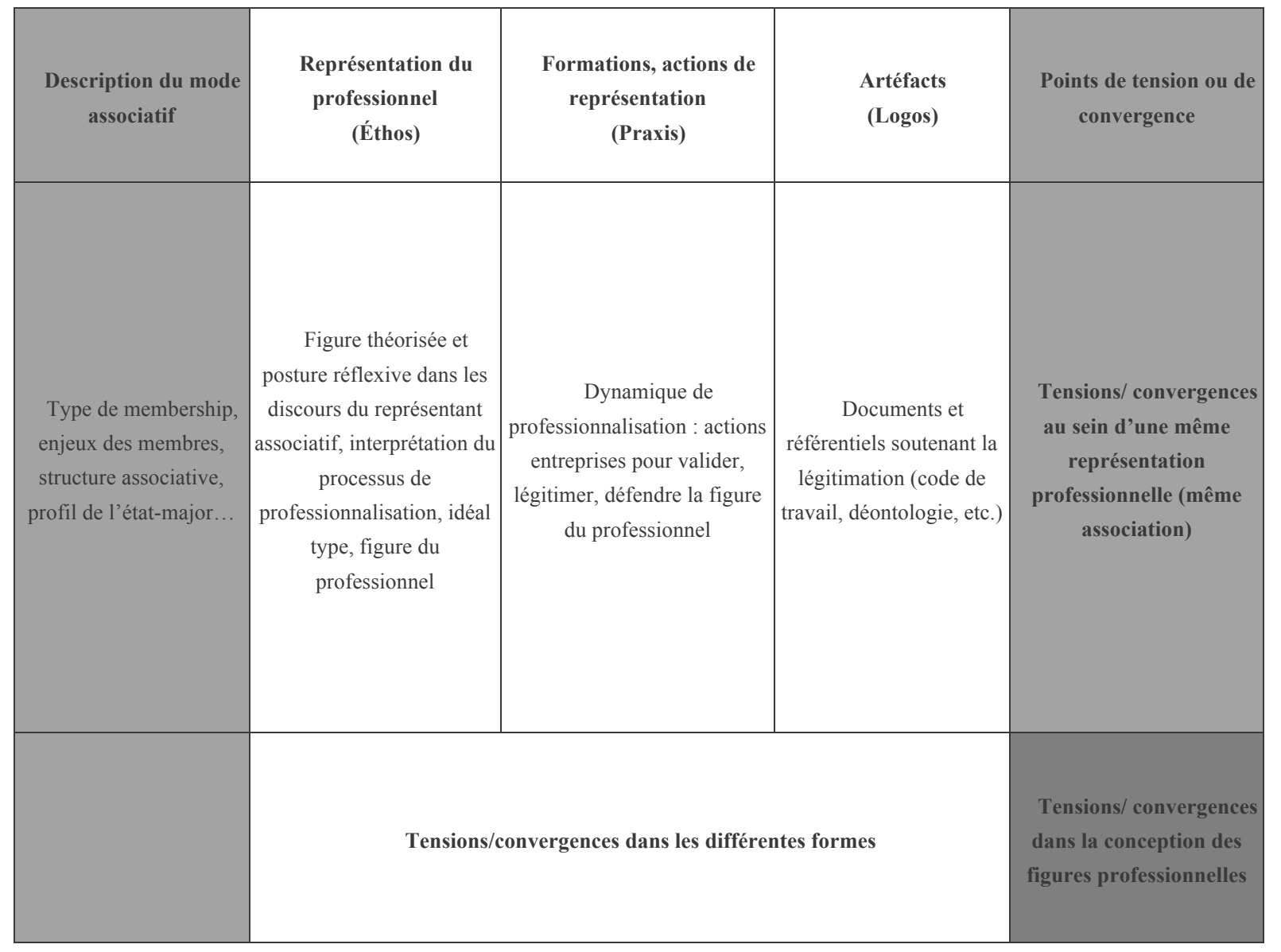


Cette matrice nous permet non seulement de relever les points de tension ou de convergence entre l'éthos, la praxis et les artéfacts - donc dans la dynamique de la professionnalisation -, mais également nous permet de voir si au sein d'une même catégorie des tensions ou des convergences sont décelables. En circonscrivant les zones de tension/convergence, la matrice permet ainsi d'identifier, pour reprendre Brown, (1989, p.165) : 1) la cohérence globale entre l'expérience des représentants et la façon dont cette expérience est exprimée ou performée; 2) la mémoire individuelle et associative démontrant une forme de continuité dans la professionnalisation des agents; 3) l'engagement conscient et réflexif des représentants, engagement leur permettant de comprendre leur position effective face à ces conceptions du professionnel de la communication.

\subsection{La mise en relation des tensions}

Les tensions ou les convergences entre les différentes formes de professionnalisation définissent en quelque sorte un spectre dans lequel s'inscrit cette professionnalisation. Par exemple, l'éthos du professionnel peut être soutenu par la maîtrise de compétences techniques (le professionnel doit savoir faire...), ou à l'inverse être présenté comme étant d'abord un professionnel doté d'une vision stratégique hors des techniques. La dernière étape a donc consisté à produire des cartes conceptuelles qui permettent d'avoir un regard global sur les points de convergence et de tension repérés dans les matrices pour l'ensemble des associations.

Pour chaque forme (éthos, praxis, artéfact), nous avons dégagé des axes servant d'abscisses et d'ordonnées. Nous avons défini les axes suivants :

- l'éthos professionnel en fonction de 1) la position dans l'organisation, selon qu'elle est orientée «tâche» ou orientée «stratégique »; 2) la posture, s'appuyant sur une pragmatique d'efficacité ou des valeurs déontiques;

- la praxis, comme agir institutionnel 1) pour doter les professionnels d'outils méthodologiques ou conceptuels; 2) pour représenter les membres auprès de la communauté des professionnels ou en regard des parties prenantes externes;

- les artéfacts comme le résultat des dispositifs 1) outillant l'activité de manière normative : « voici comment faire, comment agir » ou réflexive : « qu'avonsnous à dire sur ces pratiques $»$; 2) ayant une intention autoréférentielle ou promotionnelle.

Puis, nous avons procédé à une organisation thématique (Attride-Stirling, 2001). Nous avons regroupé, sur de grands tableaux, des unités de contenus selon qu'ils possèdent des dénominateurs communs (Figure 1). 


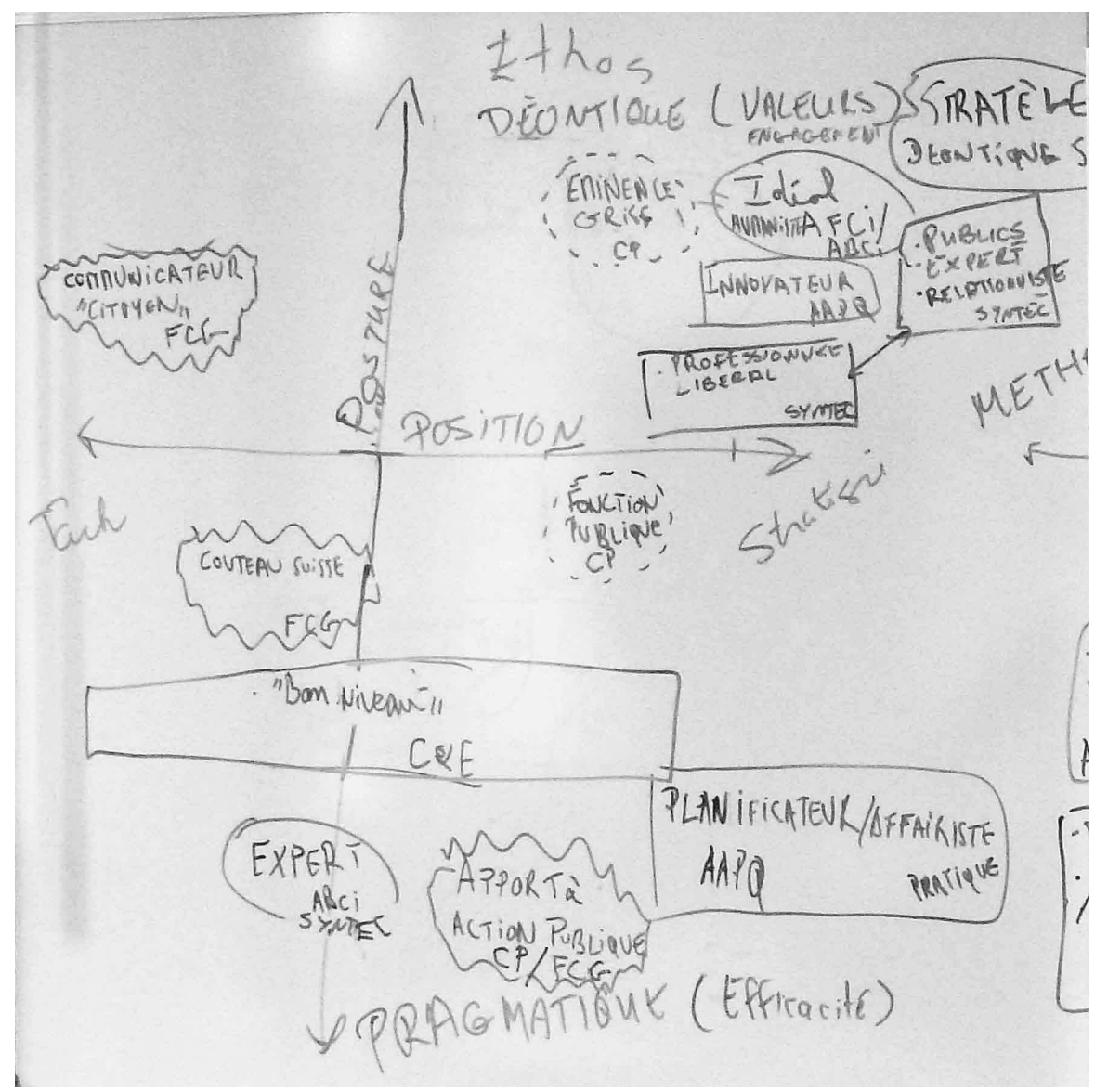

FIGURE 1

Processus de catégorisation des matériaux de base 
$\mathrm{Au}$ fur et à mesure du processus, des catégories ont commencé à émerger fédérant ainsi les unités entre elles (Figure 2). Ces différentes catégories sont explicitées dans le tableau 2 ci-après.

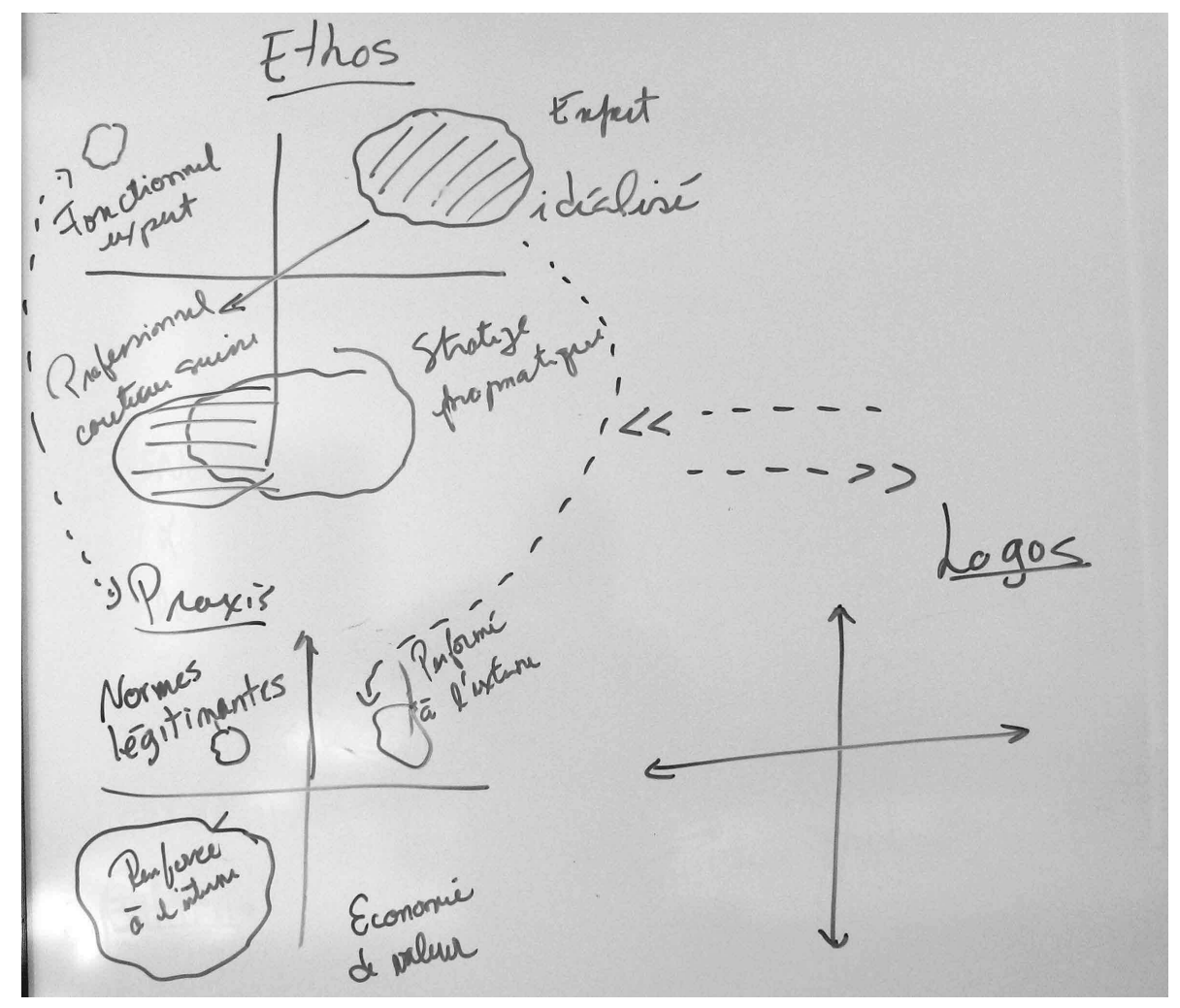

FIGURE 2

Processus de thématisation 


\section{TABLEAU 2}

\section{Définition des catégories}

\begin{tabular}{|c|c|c|}
\hline Forme & Nom de la catégorie & Explication \\
\hline \multirow[t]{4}{*}{ Éthos } & $\begin{array}{l}\text { Professionnel } \\
\text { « couteau suisse » }\end{array}$ & Exécutant polyvalent centré sur les moyens. \\
\hline & Stratège expert & $\begin{array}{l}\text { Exécutant polyvalent, mais qui justifie son expertise en } \\
\text { utilisant des indicateurs de performance pour justifier ses } \\
\text { tactiques. }\end{array}$ \\
\hline & Expert fonctionnel & Mobilise sa valeur professionnelle dans sa pratique. \\
\hline & Expert idéalisé & $\begin{array}{l}\text { Stratège dont la légitimité est basée sur un discours } \\
\text { déontique (valeurs, humanisme, innovation, dialogue } \\
\text { interne). }\end{array}$ \\
\hline \multirow[t]{4}{*}{ Praxis } & $\begin{array}{l}\text { Valorisation } \\
\text { l'externe }\end{array}$ & $\begin{array}{l}\text { Production de la figure par adossement sur le monde } \\
\text { extérieur (universitaires, experts externes). }\end{array}$ \\
\hline & Normes légitimantes & $\begin{array}{l}\text { Certifications, agrégation et formations témoignant du } \\
\text { professionnalisme. }\end{array}$ \\
\hline & Création de valeur & $\begin{array}{l}\text { Valorisation économique de la plus-value intellectuelle du } \\
\text { professionnel (mission commerciale de l'association, } \\
\text { relations avec les parties prenantes). }\end{array}$ \\
\hline & $\begin{array}{l}\text { Renforcement } \\
\text { l'interne }\end{array}$ & $\begin{array}{l}\text { Mises à l'épreuve à l'interne de l'association } \\
\text { (autoformation, retour sur des cas d'école) }\end{array}$ \\
\hline \multirow[t]{4}{*}{ Artéfacts } & $\begin{array}{l}\text { Cristallisation } \mathrm{du} \\
\text { professionnalisme }\end{array}$ & $\begin{array}{l}\text { Production d'artéfacts stabilisant et structurant le } \\
\text { professionnalisme (prix, trophée, charte, texte fondateur). }\end{array}$ \\
\hline & Vitrine identitaire & $\begin{array}{l}\text { Mise en scène et mise en acte d'une réflexion sur l'identité } \\
\text { du professionnel (livre, site Internet promotionnel). }\end{array}$ \\
\hline & $\begin{array}{l}\text { Élaboration } \mathrm{du} \\
\text { professionnalisme }\end{array}$ & $\begin{array}{l}\text { Utilisation des ressources informationnelles de façon à faire } \\
\text { évoluer le professionnalisme (benchmarking, recensement } \\
\text { des «bonnes » et « mauvaises » pratiques) }\end{array}$ \\
\hline & Veille identitaire & $\begin{array}{l}\text { Production de ressources informationnelles permettant } \\
\text { d'interroger et d'actualiser l'identité du professionnel } \\
\text { (classement des entreprises, évolution des métiers, } \\
\text { baromètres des métiers). }\end{array}$ \\
\hline
\end{tabular}


Durant ce processus, nous avons dénombré la quantité d'éléments formant chaque catégorie, de façon à pondérer l'importance de cette catégorie dans le discours des représentants. Leur importance est conséquemment représentée dans la taille des agrégats de notre carte perceptuelle (voir ci-après).

Cette production de sujets, catégories et thèmes suit la proposition d'AttrideStirling (2001) sur l'organisation thématique et permet de produire inductivement du sens avec des matériaux qualitatifs. De plus, la présence de plusieurs chercheurs au moment de l'organisation thématique permet une «triangulation d'investigation » (Patton, 2002) où la diversité des points de vue assure une plus grande fiabilité des catégories. Dans la présente étude, sept chercheurs ont contribué à définir ces catégories.

\section{Analyse et interprétation des premiers résultats}

\subsection{Constat de tensions communes aux associations}

Malgré l'hétérogénéité présente à priori dans les entrevues, notre analyse, au travers du cadre proposé, témoigne d'une part d'une congruence dans les représentations de la professionnalisation et d'autre part d'une cohérence dans les tensions communes à l'ensemble des associations rencontrées. L'analyse comparative a permis l'émergence de figures que nous avons ensuite placées sur des cartes conceptuelles qui font clairement apparaître les tensions qui existent entre ces trois moments performatifs des figures professionnelles.

Comme il est illustré dans le schéma ci-dessous, nous dégageons trois tensions (numérotées de un à trois dans la figure ci-dessous) au cœur de la professionnalisation :

- Tension 1: la part prépondérante des figures du professionnel «couteau suisse » et du stratège expert, soutenue par une praxis de renforcement des pratiques à l'interne par rapport à d'autres agrégats qui témoignent d'une aspiration à une professionnalisation déontique d'une part, stratégique d'autre part;

- Tension 2 : la tension entre l'idéal véhiculé par les associations et une constante polarisation vers la tâche des professionnels: entre l'expert idéalisé et le professionnel « couteau suisse »;

- Tension 3 : un apparent débalancement entre la grande quantité d'artéfacts normatifs (livret, code déontologique, certifications) qui contribue à la cristallisation du professionnalisme et leur réelle performativité dans la praxis et dans l'éthos. 


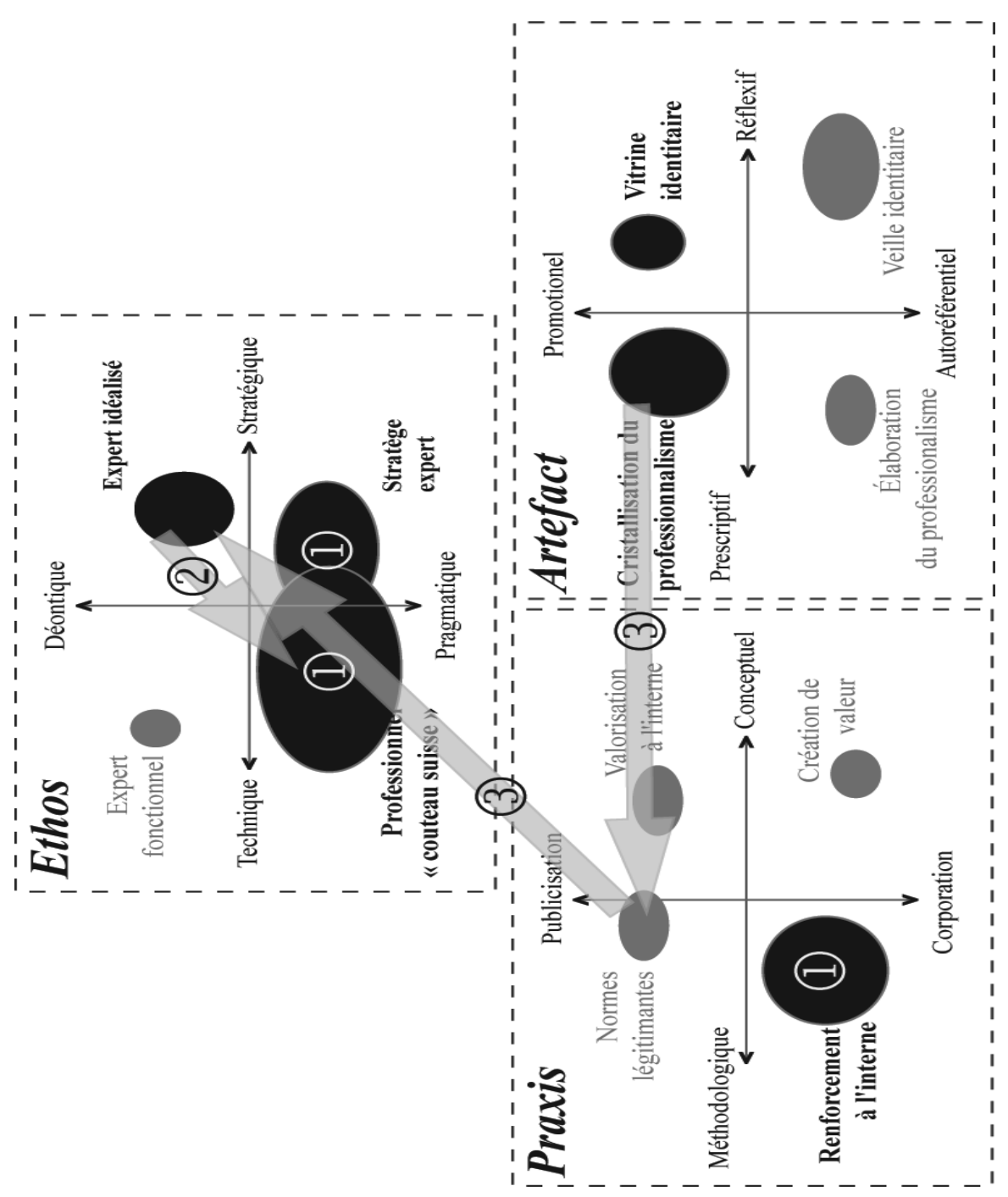

FIGURE 3 
Dynamiques de tension dans la professionnalisation

\subsection{Explicitation des trois tensions majeures}

\section{Tension 1 : Aspiration à une professionnalisation déontique et stratégique}

L'émergence de cette tension est liée au travail d'agrégation des matériaux que nous avons placés sur les cartes conceptuelles. Au niveau de l'éthos, la tension se manifeste à travers le déséquilibre dans les poids attribués ${ }^{10}$ à certaines figures : en l'occurrence, la prédominance de la figure du professionnel couteau suisse, au détriment d'autres professionnalités envisagées ou souhaitées.

«[...] dans le monde des professionnels de la communication interne, vous avez un énorme écart, en fait, entre les praticiens. Vous avez des gens, et ils sont peu nombreux et c'est à eux que je fais allusion, qui ont pu aller jusqu'au comité de direction, qui ont pu aller là où se prennent les décisions stratégiques et c'est une poignée de gens, c'est très peu [...]; vous avez le plus grand nombre qui est dans un rôle d'exécution. [...] sans aucun pouvoir hiérarchique » (ABCI).

Concernant la praxis, l'activité associative est d'une part, tournée vers la promotion et la valorisation à l'externe de la communication comme productrice de sens et de valeurs (tant morales qu'économiques). D'autre part, elle est très fortement orientée vers les actions d'autoformation avec un développement important de sessions d'apprentissage destinées aux membres/adhérents professionnels.

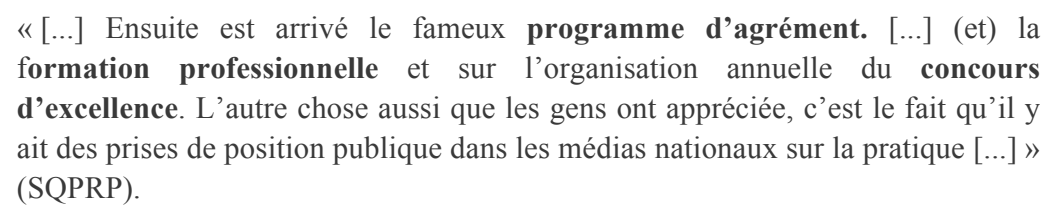

On retrouve cette orientation dans les artéfacts, notamment dans la production de guides ou d'outils équipant le professionnel :

«On édite un magazine [...] avec des sujets issus de nos différents comités de travail, soit sur des sujets happés dans l'actualité, sur des questionnements, de nos adhérents sur des thématiques précises. [...] On en refait un dans trois

\footnotetext{
${ }^{10}$ Ce poids résulte de la convergence dans la catégorisation des matériaux discursifs.
} 
semaines, avec la directrice de la communication qui était en poste chez France Télécom quand toute cette vague de suicides est arrivée. Elle va venir nous expliquer comment on gère la crise et comment on met en place derrière tous les outils de communication et toute la stratégie, qui va aider à passer le cap interne, voilà on va avoir des sujets comme cela.» $(\mathrm{C} \& \mathrm{E})$

En ce sens, cette première tension provient du renforcement de cette figure performée dans l'éthos, la praxis et les artéfacts qui pèse par la place qu'elle prend sur le développement d'autres figures.

\section{Tension 2 : Entre un idéal véhiculé par les associations et une constante polarisation vers la tâche des professionnels}

L'analyse des figures du professionnel de la communication dans la construction de l'éthos révèle une tension entre, d'une part, l'aspiration à se positionner éthiquement sur un ensemble de valeurs humanistes, de dialogue interne, d'innovation organisationnelle soutenant une expertise stratégique.

«Il y a un très haut niveau d'expertise [...] on a l'obligation de mettre en face ce qu'on dit et ce qu'on fait autour de valeurs » (Syntec RP)

Et d'autre part, le constat d'un vécu de praticien fonctionnel, polyvalent, véritable « couteau suisse » à la fois modeste et virtuose de la production des outils ou des dispositifs auxquels les impératifs tactiques de la communication l'appellent.

"C'est un peu comme un couteau suisse : faire la démonstration de toutes les utilités qu'un communicateur ou une communicatrice peut poser comme gestes avec extrêmement de rigueur. » (FCG)

« Dans communication, il y a un mot essentiel, c'est "l'autre" et il y a l'idée de la relation et cela veut dire que se définir par les outils c'est réducteur [...] on est lucide, le métier c'est encore beaucoup la mise en oeuvre d'outils, on n'est pas encore sur une vision de la com' qui est axée sur la relation » (AFCI)

Cette tension est vécue au sein des organisations, certes, mais également entre les attentes des membres des associations et la direction de ces associations.

«[Les membres] ne sont pas contents de payer pour que je fasse ces activités-là [démontrer aux parties prenantes le potentiel créatif des membres]. C'est très théorique, ça paraît bien dans les conversations, mais les gens veulent que leur association fasse des choses vraiment concrètes [...] Négocier les ententes collectives, régler les problèmes de l'industrie (les mauvais appels d'offres)... » (AAPQ) 


\section{Tension 3 : Un apparent débalancement entre les artéfacts normatifs et leur réelle performativité dans la praxis et dans l'éthos.}

Nous pointons une troisième tension résultant de la production par les associations d'un nombre important d'artéfacts (ex. livrets, codes déontologiques...) destinés à « cristalliser» le professionnalisme. Ces nombreux artéfacts ne sont pas mobilisés dans la praxis associative, ni semble-t-il dans les pratiques professionnelles des adhérents. Ainsi, même s'ils témoignent de la volonté des associations d'asseoir un idéal professionnel pour le communicateur, leur performativité n'est pas présente dans la praxis.

«Un code de déontologie c'est basé sur voici comment on va faire les choses dans notre organisation. Dans le cas actuel de la SQPRP, c'est une suggestion pour les gens qui adhèrent. La faiblesse de tous ces codes-là c'est que les intentions et les contenus sont très bons, mais tu n'as jamais la police pour le faire appliquer. Ça coûterait très cher de le faire. » (SQPRP)

«En 1998, nous avons voté, nous avons écrit, notre charte de déontologie et nous l'avons votée à la quasi-unanimité. C'est important cette charte de déontologie parce qu'elle au fond inspirée du code de déontologie des journalistes professionnels, mais elle n'a de force et d'impact que sur nous-mêmes, elle est autoproclamée, elle n'a jamais été reconnue par aucune autre instance, ni gouvernementale, ni d'entreprise [...] S'il y a manquement... il y a simplement le fait que nous déplorons publiquement que quelqu'un ne respecte pas les principes du code $»(\mathrm{ABCI})$.

\section{Discussion et conclusion}

\subsection{Des tensions explicitées aux tensions expliquées}

Dans cette dernière partie, nous tentons d'émettre trois hypothèses pouvant expliquer ces tensions. Une première explication réside dans la prééminence du système de valeurs - efficacité, efficience et performance — omniprésent dans les entreprises. Elle implique que les responsables de communication doivent aujourd'hui planifier leurs actions, quantifier leurs objectifs et les évaluer de façon permanente (Le Moënne, 1993) sans quoi ces activités de communication risquent bien souvent de tomber dans la case " gaspillage » face à la recherche constante des indicateurs de ROI (retour sur investissement) et des baisses de coûts. Cette tension se retrouve notamment dans l'obsession dans la production (Heller, 2001) des 
associations qui mettent à la disposition des membres des brochures ou guides de production et d'évaluation de communication ${ }^{11}$.

Une seconde hypothèse est celle de la croissance de la technicité du travail de communication. Certaines études (voir l'article de Valérie Lépine dans ce même numéro) témoignent d'une augmentation de la dimension technique du métier au détriment de son aspect plus réflexif et éthique. L'explosion d'Internet n'est certainement pas étrangère au bouleversement que connaissent les codes et les pratiques de communication.

Une troisième hypothèse, résultante des deux premières, est le rapport déficient au temps chez le professionnel. Mis sous pression constante tant d'un point de vue technique que managérial, le professionnel devient le producteur de ces tensions et de leur renforcement parce qu'il n'a peu ou prou l'opportunité de prendre du recul par rapport à la tâche.

En tant que réseau portant une réflexion sur les phénomènes de professionnalisation, nous poursuivrons notre travail en testant et en éprouvant ces hypothèses que ce soit auprès des associations ou des professionnels eux-mêmes.

\subsection{Implications pour les associations professionnelles}

La pluralité des figures et les tensions identifiées montrent combien le tiraillement entre impératifs de performance quantitative et conscience d'une complexité de l'organisation et de l'humain traverse la question de la professionnalisation. Il est difficile d'en faire grief aux associations représentant des membres en quête de légitimité dans un espace où les règles du jeu sont déterminées par d'autres. Les associations n'ont, en effet, que peu de marges de manœuvre.

Néanmoins, nous pensons que ce miroir tendu aux associations ainsi que la méthode proposée ici pourraient servir comme un outil d'aide à la mise en place de politiques associatives. Pratiquement, les représentants associatifs pourraient se livrer régulièrement à cet exercice visant à confronter éthos, praxis et logos. Ils pourraient également confronter les résultats aux discours et pratiques de leurs membres. Ils ouvriraient ainsi un débat interne propice à une réflexion collective entre professionnels de la communication.

\footnotetext{
${ }^{11}$ Par exemple, le COMNet, association fédérale Belge des communicateurs actifs dans les ministères propose une collection très orienté dans ce sens http://www.fedweb.belgium.be/fr/a_propos_de_1_organisation/communication/a_propos_de_la_co mmunication_federale/reseaux/commnet/
} 


\section{Bibliographie}

Abbott, A. (1988). The System of Professions : An Essay on the Division of Expert Labor, Chicago : University of Chicago Press.

Attride-Stirling, J. (2001). Thematic networks: an analytic tool for qualitative research. Qualitative Research, 1(3), 385-405. doi:10.1177/146879410100100307

Brown, R. H. (1989). Social science as civic discourse: Essays on the invention, legitimation, and uses of social theory. University of Chicago Press.

Brulois V., J-M. Charpentier (2010). Les métiers de la communication en entreprise face au défi de la connaissance, Actes du 17e colloque de la SFSIC.

Callon, M., Latour, B., \& Akrich, M. (2006). Sociologie de la traduction : textes fondateurs, Paris : Presses de l'Ecole des Mines.

Caldwell, J. T. (2006). Cultural Studies of Media Production: Critical Industrial Practices. In M. White \& J. Schwoch (Eds.), Questions of method in cultural studies (pp. 109-153). Malden: Wiley-Blackwell.

Champy, F. (2011). Nouvelle théorie sociologique des professions, Paris: Presses Universitaires de France - PUF.

Chapoulié, J.-M. (1973). Sur l'analyse des groupes professionnels, Revue française de sociologie, 14 (1), 155-244.

Cooren, F. (2010a). Action and Agency in Dialogue: Passion, Ventriloquism and Incarnation, Philadelphie : John Benjamins Publishing Company.

Cooren, F. (2010b). Ventriloquie, performativité et communication. Ou comment fait-on parler les choses, Réseaux, 163 (5), 33-54.

Cooren, F. (2010c). Comment les textes écrivent l'organisation. Figures, ventriloquie et incarnation. Études de communication, 34(1), 23-40.

de la Broise, P. (2006). La professionnalisation des communicateurs : De la fonction aux métiers, Education permanente, (167), 67-78.

de la Broise, P., Chantraine, O., Huet, R. (2008). La professionnalisation de la responsabilité : dynamiques, formes et figures professionnelles du Développement Durable $\quad[\mathrm{PDF}]$ Repérée à http://www.meshs.fr/documents/pdf/seminaires/2009/DelaBroise_Huet.pdf

Dubar, C., Tripier, P. (2005). Sociologie des professions $\left(2^{\mathrm{e}}\right.$ édition revue et augmentée.), Paris : Armand Colin. 
Eisenberg, E. M., \& Riley, P. (2001). Organizational Culture, dans F. M. Jablin \& L. Putnam (Dirs.). The new handbook of organizational communication: Advances in theory, research, and methods, Thousand Oaks : Sage Publications, 291-322.

Gaulejac, V. de. (2005). La société malade de la gestion : Idéologie gestionnaire, pouvoir managérial et harcèlement social, Paris : Seuil.

Geertz, C. (1973). The interpretation of cultures: Selected essays, New York : Basic books.

Grant, D., Hardy, C., Oswick, C., \& Putnam, L. (2004). The Sage handbook of organizational discourse, London : Sage Publications Ltd.

Heller, T. (2001). Management symbolique et communication - l'argument de l'histoire, Communication et organisation, 20. Repéré à http://communicationorganisation.revues.org/2597.

Hesmondhalgh, D., \& Baker, S. (2011). Creative Labour. New York : Routledge.

Huet, R., de la Broise, P. (2010). La responsabilité sociale des entreprises en quête d'auteurs, in Communication et Organisation, 37 (1), 65-77.

Jackson, T. L. (1996). Implementing a Lean Management System, Portland: Productivity Press.

Joris, G., \& Fallon, C. (2009). L'administration dans un contexte de modernité radicale : quand les instruments de gestion doivent devenir source de réflexivité, Pyramides. Revue du Centre d'études et de recherches en administration publique, 18, 55-72.

Jorro, A. (2009). La construction de l'éthos professionnel en formation alternée. Travail et apprentissage, (3), 13-25.

Latour, B. (1989). La science en action, Paris : La Découverte.

Le Moënne, C. (1994). Communication « by smiling around » et crise managériale, Réseaux, (12), 29-52.

Lépine, V., Brulois, V., Lambotte, F., Coyette, C., David, M. D., Baillargeon, D., De La Broise, P., Bessières, D., Chauvin, D. (9 mars 2012), De la reconnaissance mutuelle et publique des professionnels de la communication, Actes du colloque Communiquer dans un monde de normes, Roubaix.

Martin, J. (2002). Organizational culture: Mapping the terrain. London: Sage Publications, Inc.

Mintzberg, H. (1983). Structure in Fives, Designing Effective Organizations, Englewood Cliffs, Prentice-Hall. 
Moënne, C. L. (1993). Utopies du troisième type. Communications managériales et utopisme, in Mots, vol 35, no 1, p. 86-108.

Patton, M. Q. (2002). Qualitative research \& evaluation methods (3rd ed.). Thousand Oaks, CA : Sage.

Ricoeur, P. (1996). Soi-même comme un autre, Paris : Seuil.

Trujillo, N. (1983). "Performing" Mintzberg's roles: The nature of managerial communication, in L. Putnam \& M. E. Pacanowsky (Eds.), Communication and organizations, an interpretive approach, Newbury Park, Sage Publications, Inc, p. 73-97.

Rochet, C., Keramidas, O., Tiberghien, B., Agopian, P., \& Paris-Laporte, C. (2010). Management des services publics et pilotage de la performance: vision en contre-plongée de la Loi d'Orientation sur les Lois de Finance, Pyramides. Revue du Centre d'études et de recherches en administration publique, (19), p. 203-218.

Scieur, P. (2011). Sociologie des organisations, Paris : Armand Colin.

Silverman, D. (2000). Doing qualitative research. A practical guide. London : Sage.

Strauss, A. L. (1991). La trame de la négociation: Sociologie qualitative et interactionnisme, Paris : L'Harmattan.

Vásquez, A. J. et C. (2011). Reconfiguration de l'organisation : suivre à la trace les figures textualisées - le cas de la figure du patient. Études de communication, $n^{\circ}$ 36(1), 129-146.

Weick, K. E. (1995). Sensemaking in Organizations. London : Sage Publications 